\title{
A High Fiber Diet Responsive Case in a Poodle Dog with Long-Term Plant Eating Behavior
}

\author{
Byeong-Teck KANG ${ }^{1)}$, Dong-In JUNG ${ }^{1)}$, Jong-Hyun $\mathrm{YOO}^{2)}$, Chul PARK ${ }^{3)}$, Eung-Je $\mathrm{WOO}^{4)}$ and Hee-Myung PARK ${ }^{1) *}$ \\ ${ }^{1)}$ Department of Veterinary Internal Medicine and ${ }^{21}$ BK21 Program of Integrative Network Systems for Veterinarians in Basic Science \\ Industrial Animals and Preventive Medicines, College of Veterinary Medicine, Konkuk University, \#1 Hwayang-dong, Gwang-jin-gu, \\ Seoul 143-701 ${ }^{3)}$ Acupuncture \& Meridian Science Research Center and ${ }^{4}$ College of Electronics and Information, Kyunghee University, 1 \\ Seocheon-dong, Giheung-gu, Yongin-si, Gyeonggi-do 446-701, South Korea
}

(Received 15 November 2006/Accepted 29 March 2007)

ABSTRACT. An 11-year-old, castrated male Miniature Poodle dog was referred due to daily plant eating followed by vomiting for 7 years. Each time the dog went out for a walk, he had been munched grass, and then vomited. There were no abnormal findings on medical examinations. Through behavior examination and consultation, we diagnosed this dog as having a plant eating problem. Because we assumed that the dog had eaten plants to supply of dietary deficiency or relieve digestive problems, advised the owner to discontinue supplying previous diet and to feed high-fiber diet. On the follow up, the owner said that the dog had not eaten plants and vomited from 3 days after initial dietary change. The dog has not shown any clinical signs for 13 months. This clinical finding shows a possibility that deficient diet, especially fiber, relates to the plant eating behavior of a dog.

KEY WORDS: high-fiber diet, plant eating, Poodle dog.

J. Vet. Med. Sci. 69(7): 779-782, 2007

The dog (Canis familiaris) was the first animal domesticated by human beings, as dogs have been living with humans for at least 14,000 years (possibly even longer) [6, 17]. Many morphological, behavioral, genetic studies have confirmed the idea that dogs are the direct descendants of wolves (Canis lupus) [16-18]. The wolf is carnivorous, feeding on most of game from large ungulates such as elk to small rodents like mice. However, it is shown that 2 to $10 \%$ of feces and stomach contents of wild wolves was plants, especially grass [1]. Feces of many wild carnivores also contain a considerable amount of vegetable matter [7].

Dogs are more omnivorous than wolves. Although dogs belong to the mammalian order Carnivora, they are widely believed to be omnivorous in dietary habits and digestive capabilities [5]. Ingestion of plants, including grasses, fruits and vegetables, can be commonly watched in dogs. However, no one exactly knows why dogs eat plants. Although there are several explanations that have been offered for this behavior based on anecdotal evidence, these theories were not scientifically validated or approved because there have been few researches into the plant-eating behavior of domestic dogs [14].

This note describes a case of daily plant eating followed by vomiting in a Poodle dog, which had continued for 7 years and was instantly ceased when high-fiber diets was only provided. It is a first case report of plant eating problem in a dog.

An 11-year-old, castrated male Miniature Poodle dog (body weight: $6 \mathrm{~kg}$ ) was referred due to the behavior prob-

\footnotetext{
* Correspondence to: Park, H.-M., Department of Veterinary Internal Medicine, College of Veterinary Medicine, Konkuk University, \# 1 Hwayang-dong, Gwang-jin-gu, Seoul 143-701, South Korea

e-mail: parkhee@konkuk.ac.kr
}

lem relating to inappropriate item ingestion for 7 years. The household consisted of a husband and wife, their three daughters aged with 25-, 28-, 31-year-old, respectively. The dog had been obtained at 3 months of age from a near relation. One month after adaptation to new housing environment, the walk with the dog had been taken at least once a day. Usually, it was regularly taken with daughters in a public garden near the house at about 4 in the afternoon. When the dog was 4 years old, he had intermittently munched grass in a public garden, and then vomited during the walk. Six months after abnormal behaviors were firstly noted, those had been continuously shown each time went out for a walk. The places the dog ate grass and vomited were daily different. On a day the walk could not be taken, the dog ate plants, such as orchid and grass, grown in house, and then vomited whether the owners present or not. Specific behaviors patterns immediately preceded and followed the planteating behavior were not noted except for vomiting. The frequency of plant eating was only one per a day. To distract or interrupt the dog from plant eating, none managements or punishments had not been taken by the owners. Until the admission, this plant eating and vomiting had been daily repeated. The status of feces had been mildly flaccid and soft. The dog had been kept indoors without other animals, regularly received all vaccinations (rabies, canine distemper, hepatitis, parvovirus, parainfluenza, and leptospirosis) and anti-helminthics, and supplied with commercial dry food. In addition, he tested negative for heartworm infection in local animal hospital before the admission. At the presentation day, the dog was previously fasted for $12 \mathrm{hr}$ to examine medical tests.

Physical examinations revealed that the dog was alert, obese (body condition score: 4), and had dry skin. Abnormalities on the complete blood count (CBC), serum bio- 
chemical profile, and urine analysis were not remarkable. There were no remarkable findings on neurological, abdominal radiographic and ultrasonographic examinations. A fecal flotation was negative for parasitic ova, and fecal cytology was normal. Reverse transcription polymerase chain reaction (RT-PCR) for canine distemper and parvo virus tested negative. To help evaluate gastrointestinal (GI) functions (digestion and absorption) and rule out medical causes (e. g. small intestinal bacterial overgrowth, inflammatory bowel disease, neoplasia) for the abnormal eating and chronic vomiting, various tests were examined. Serum cobalamin, folate, trypsin-like immunoreactivity (TLI), thyroxine $\left(\mathrm{T}_{4}\right)$, amylase, and lipase levels were within normal range. No fat globules were found in fecal stain with Sudan III. Results from the fecal culture were negative for Salmonella spp., Campylobacter jejuni, and Clostridium spp., but Streptococcus spp. and Escherichia coli were isolated. The latter were not considered pathogenic. The results of all physical examination and laboratory tests are summarized in Table 1. Because any abnormalities were not found in laboratory examinations, endoscopy was executed. Macroscopically, GI tracts appeared normal. Multiple biopsies of stomach and intestine were taken. Histological examination was within normal limits. Because gastrointestinal signs like vomiting and diarrhea are also noted on food allergy and any abnormalities were not detected on various tests, feed the dog a commercially prepared food (Prescription Diet Canine $\mathrm{z} / \mathrm{d}^{\circledR}$, Ultra allergen-free, Hill's pet nutrition) as a hypoallergenic test diet for 8 weeks. However plant eating and vomiting were not disappeared and continued. Based on the age of onset of clinical signs, no pruritus and cutaneous lesions (e. g. papules, erythema, epidermal collarettes, pododermatitis, seborrhea, and otitis externa), normal hemogram, and none clinical improvement for hypoaller- genic test diet, food allergy could be ruled out.

The differential diagnoses for plant eating behavior that was considered included attention seeking behavior, reinforced behavior, situational anxiety, and behaviors associated with compulsive disorders, such as conflict- and frustration-induced behaviors, and displacement behavior. Congenital, infectious, metabolic, neurological, and hormonal diseases that could contribute to pica and vomiting were also considered but were ruled out on the basis of the normal physical, neurological examinations and laboratory testing results. Attention seeking and reinforced behavior were ruled out by the absence of owners' specific responses (e. g. attention, compensation, and punishment) to the plant eating behavior of dog and the occurrence of it both in and outside of the presence of the owners. Compulsive disorders were considered because of the pica, which is the one of expression of compulsive disorders in dogs, and the stereotypic feature of the behavior. However, those were ruled out because the causes of compulsive disorders, which can lead to anxiety, stress, conflict, and frustration, could not found on the behavior consultation and examinations. Also other clinical signs related to compulsive disorders, such as locomotory, hallucinatory, self-injurious or self-directed signs, were not noted at all. The dog had been regularly exercised, greatly enjoyed walking with the owners, and friendly interacted with other people and animals during the walk. There were no fixed area where abnormal behaviors were noted and specific situations, such as noises and fireworks. Based on these histories, it was thought that specific environments and anxiety were not related to plant eating behavior. Other behavior problems as well as coprophagia and stone chewing were also not noted. Thus we tentatively diagnosed this dog as having truly a plant eating behavior problem.

Table 1. The results of physical examination and laboratory tests

\begin{tabular}{|c|c|c|c|}
\hline & Examination & Result & Reference range \\
\hline Physical & Mental status & alert & - \\
\hline \multirow[t]{2}{*}{ examination } & Body condition score & 4 & 3 \\
\hline & Skin & Dry skin & - \\
\hline \multirow[t]{14}{*}{ Laboratory tests } & Complete blood count & NRF & - \\
\hline & Serum biochemical profile & NRF & - \\
\hline & Urine analysis & NRF & - \\
\hline & Fecal flotation \& cytology & NRF & - \\
\hline & Fecal culture & $\begin{array}{c}\text { Streptococcus spp. } \\
\text { Escherichia coli }\end{array}$ & - \\
\hline & Fecal stain with Sudan III & NRF & \\
\hline & CDV (RT-PCR) & negative & - \\
\hline & CPV (RT-PCR) & negative & - \\
\hline & Cobalamin $(\mathrm{pg} / \mathrm{m} l)$ & 360 & $175-550$ \\
\hline & Folate $(n \mathrm{~g} / \mathrm{m} l)$ & 10 & $6.7-17.4$ \\
\hline & $\operatorname{TLI}(\mu \mathrm{g} / l)$ & 15 & $5-35$ \\
\hline & Thyroxine $\left(\mathrm{T}_{4}\right)(\mu \mathrm{g} / \mathrm{d} l)$ & 2.5 & $1.0-3.5$ \\
\hline & Amylase $(\mathrm{U} / l)$ & 710 & $220-1070$ \\
\hline & Lipase $(\mathrm{U} / l)$ & 250 & $90-527$ \\
\hline
\end{tabular}

CDV: Canine distemper virus; CPV: Canine parvo virus; TLI: Trypsin-like immunoreactivity; NRF: No remarkable finding. 
Generally, specific causes and treatments of plant eating behavior had not been found. Thus, we assumed that the dog had eaten plants to supply of dietary deficiency or relieve digestive problems as he had repeatedly vomited after eating plants. We advised owner to discontinue supplying previous diet and to feed high-fiber diet. The recommended diet was a commercial prescription diet (contains $15.3 \%$ crude fiber) (Prescription Diet Canine w/d ${ }^{\mathbb{Q}}$, Hill's pet nutrition) with cooked or raw grains and vegetables, such as bran, soy bean, and beet pulp. The total estimated proportion of fiber in diet was adjusted to $20 \%$. Diet change was gradually made in $15 \%$ per day over 1 week. Caloric intake was maintained at $70 \mathrm{kcal} / \mathrm{kg}$ per day.

The response of the dietary change was evaluated by questioning the owner by telephone at 5 days after the initial change. The owner said the dog had not eaten plants and vomited from 3 days after the initial change. One week after the dog had been eating solely the high-fiber diet, the dog was revisited to animal hospital and had no significant changes in body weight and hemogram. According to the owner's explanation, feces had been solidified and bulk was increased in proportion with the rate of diet change. The owner was very satisfied with disappearing behavior problems. After consultation, the dog was regularly monitored by telephone. Until recently, the dog that fed with a highfiber diet for 13 months has not shown any clinical signs such as plant eating and vomiting. Thus we definitively diagnosed the dog as having a plant eating behavior problem because the dog responded solely to the dietary change without behavior modifications and medications for other behavior problems and other medical problems were previously ruled out through various examinations.

There are several theories that can explain canine planteating behavior. Dogs occasionally eat plants, especially grasses, and then vomit. This has been interpreted as canine self-medication of GI distress [10]. Some dogs may ingest plants when they have an upset stomach. It is believed that vomiting after eating plants is the way dogs relieve gas pressure and other stomach upsets [10]. Bothma (1966) reported that the cape foxes intentionally eat grass during periods of prolonged starvation to keep their digestive system functioning. Plant eating may also reflect an instinctive behavior acquired from wolf ancestors among whom plant eating has been observed [14]. In addition, taste preference for plants was presented as an explanation of plant eating [11].

In this case, daily repeated behavior of plant eating and vomiting ceased after a dietary change from a commercial dry food to high-fiber diet. Because he had no abnormalities on hemogram and GI related examinations, it is thought that the dog had normal digestive and absorptive functions of small intestine and other organs, such as pancreas and liver. Also, vomiting had not been observed after dietary change, thus plant eating may not be developed to relieve GI gas pressure and stomach upsets. Even though gastrointestinal signs of food allergy can be improved after dietary change, high-fiber diets used in this case is not used as a hypoallergenic diet and it was previously ruled out by the restricted test diet. From specific time plant eating was shown, we inferred that instinct of wolf and taste was not related to this abnormal behavior.

Other theories of plant eating are supplement of a deficient diet and behavioral problems such as pica [14]. Lund [9] assumed that the red fox ingest grass to supply additional minerals and vitamins. Overall [10] supposed that ingestion of plants indicate the dog perceives a need for more fiber in its diet. Fiber is indigestible carbohydrates and is present in all plants eaten for food, including fruits, vegetables, grains, and legumes. Inclusion of dietary fiber in canine diets has been studied for a number of years and has been shown to have beneficial effects on energy metabolism, fecal characteristics, and transit times [12,13,15]. Thus, fiber is present in almost every commercial dog food. High-fiber diet has been used for not only obesity prevention, but also fiberresponsive diseases, such as diabetes mellitus, diarrhea, hyperlipidemia, colitis, lymphangiectasia, and pancreatitis [4]. Dietary fiber is also recommended as part of the therapy of acute or chronic large intestinal disease of unknown cause or idiopathic colitis [4].

Generally, dogs can intake enough nutrients and fibers from common diets. However, this dog may specially need more fibers. In this case, necessity of fibers may be closely related to fecal status and decreased function of large intestine. The large intestine of dogs, particularly colon, is responsible for the resorption of water and salt from ileal chyme [8]. Even though large volumes of water were absorbed in jejunum and ileum (up to $75 \%$ of fluids in intestine) [3], characteristics of feces may indicate decreased function of large intestine because the absorptive function of small intestine was thought to be normal in this dog based on mild obesity, normal hemogram, and no abnormalities in various tests for absorptive or digestive function of small intestine and pancreas. Fiber retains large volumes of water and the viscous gel formed with soluble fibers prolongs intestinal transit time [4], which makes it possible to achieve appropriate stool bulk and consistency.

Thus, it is thought that plant-eating behavior of this dog was caused by deficient diet, especially fibers, and vomiting was immediately erupted after plant eating by mechanical irritation of the eaten plant for the digestive tract or acute consumption of large volume of low digestible materials even though the dog needed to supplement fiber deficiency. However, it is exactly not known how the dog can recognize his deficiency of fibers and know that plants are good sources of fibers, and why he started to eat plant at 4 years old.

Recently, Sueda et al. [14] reported that the frequency of plant eating was not related to diet as well as sex, gonadal status, breed, receipt of antihelmintic medication, or other behavior problems. However, in this case, diet had found to be closely related to plant eating.

Eating large amounts of grass and plants treated with fertilizer or herbicide can irritate the GI tract. Thus, when plant eating was daily repeated for long periods, it may be necessary to consider a behavior problem due to the dietary defi- 
ciency as a possible cause after evaluating GI lesion.

ACKNOWLEDGMENT. This work was supported by the SRC/ERC program of MOST/KOSEF (R11-2002-103).

\section{REFERENCES}

1. Andersone, Z. 1998. pp. 79-80. Proceedings of the Latvian Academy of Sciences.

2. Bothma, J. du P. 1966. Vulpes chama. Zool. Afr. 2: 205-210.

3. Burrows C. F. 1983. Vet. Clin. North Am. Small Anim. Pract. 13: $521-540$.

4. Dimski, D. S. and Buffington, C. A. 1991. J. Am. Vet. Med. Assoc. 199: 1142-1146.

5. Fahey, G. C., Merchen, N. R., Corbin, J. E., Hamilton, A. K., Serbe, K. A. and Hirakawa, D. A. 1990. J. Anim. Sci. 68: 42294235.

6. Federoff, N. E. and Nowak, M. R. 1997. Science 278: 205.

7. Hoppe-Dominik, B. 1988. Naturwissenschaften 75: 49-50.

8. Murdoch, D. B. 1996. pp. 151. Manual of Canine and Feline Gastroenterology. 1st ed., British Small Animal Veterinary Association, Cheltenham.

9. Lund, H. M. K. 1962. Papers Norwegian State Game Res.Inst.
12: 1-79.

10. Overall, K. L. 1997. pp. 266-267. Clinical Behavioral Medicine for Small Animals. 1st ed., Mosby, Philadelphia.

11. Schwartz, S. 1997. pp. 59-60. Instructions for Veterinary Clients. Canine and Feline Behavior Problems. 2nd ed., Mosby, Philadelphia.

12. Simpson, J. W. 1998. J. Nutri. 128: 2717S-2722S.

13. Simpson, J. M., Martineau, B., Jones, W. E., Ballam, J. M. and Mackie, R. I. 2002. Microb. Ecol. 44: 186-197.

14. Sueda, K. C., Hart, B. L. and Cliff, K. D. 2005. pp. 230-231. Current Issues and Research in Veterinary Behavior Meeting. Purdue University, West Lafeyette.

15. Sunvold, G. D., Fahey, G. C. Jr., Merchen, N. R., Titgemeyer, E. C., Blurquin, L. D. and Reinhart, G. A. 1995. J. Anim. Sci. 73: 1099-1109.

16. Tsuda, K., Kikkawa, Y., Yonekawa, H. and Tanabe, Y. 1997. Genes Genet. Syst. 72: 229-238.

17. Vila, C., Savolainen, P., Maldonado, J. E., Amorim, I. R., Rice, J. E., Honeycutt, R. L., Crandall, K. A., Lundeberg, J. and Wayne, R. K. 1997. Science 276: 1687-1689.

18. Zimen, E. 1981.pp. 18-34. The Wolf: A Species in Danger. 1st ed., Delacorte Press, New York. 